\title{
Atuação e avanços do profissional farmacêutico no âmbito oncológico
}

Acting and advances of the pharmaceutical professional in the oncological scope

Actuación y avances del profesional farmacéutico en el alcance oncológica

Camila Maria do Nascimento Santos ORCID: https://orcid.org/0000-0003-3796-0710 Associação de Ensino Superior do Piauí, Brasil

E-mail: camilamarisantos9892@gmail.com

José Chagas Pinheiro Neto

ORCID: https://orcid.org/0000-0002-6626-6122 Universidade Federal do Piauí, Brasil E-mail: jose.pinheiro4321@gmail.com

Ian Jhemes Oliveira Sousa

ORCID: https://orcid.org/0000-0003-1930-9828 Universidade Federal do Piauí, Brasil E-mail: ianjhemes@gmail.com

Higo José Neri da Silva

ORCID: https://orcid.org/0000-0003-2089-4147 Universidade Federal do Piaú, Brasil E-mail: higoneri@gmail.com

Jenniffer Batista Neres de Carvalho ORCID: https://orcid.org/0000-0002-8482-5995 Faculdade Mauricio de Nassau, Brasil E-mail: jennifferneres21@gmail.com

Antonia Filha Moreira de Melo ORCID: https://orcid.org/0000-0002-0690-4380 Centro Universitário Uninovafapi, Brasil E-mail: afmdemello@gmail.com

Alisson Camilo Martins Sousa

ORCID: https://orcid.org/0000-0003-4613-5114

Centro Universitário Uninovafapi, Brasil

E-mail: alissoncamilo1986@hotmail.com

Denilson de Araújo e Silva

ORCID: https://orcid.org/0000-0001-5401-3462 Centro Universitário Uninovafapi, Brasil

E-mail: bmdenilsonaraujo@outlook.com Juciara Carvalho de Oliveira

ORCID: https://orcid.org/0000-0002-2010-2587 Laboratório Central do Piauí, Brasil E-mail: kelly-rego@bol.com.br

Fabiana de Souza Santana

ORCID: https://orcid.org/0000-0002-4395-773X Faculdade das Américas, Brasil E-mail: fabianasantana6@ hotmail.com

Sônia Regina Monteiro de Azevêdo

ORCID: https://orcid.org/0000-0003-3658-918X Universidade Federal do Piauí, Brasil E-mail: soniaazevedo@ufpi.edu.br

Bianca de Sousa Leal

ORCID: https://orcid.org/0000-0002-1366-8742 Universidade Federal do Piauí, Brasil

E-mail: lealbiancasat@gmail.com

Herlem Silva Rodrigues

ORCID: https://orcid.org/0000-0001-8756-9969

Centro Universitário Santo Agostinho, Brasil

E-mail: herlemsrodrigues@gmail.com

Kelly Maria Rêgo Da Silva

ORCID: https://orcid.org/0000-0003-3070-4060

Laboratório Central do Piauí, Brasi

E-mail: kellyma.aria@gmail.com

Iluska Martins Pinheiro

ORCID: https://orcid.org/0000-0002-8160-9787

Universidade Federal do Piauí, Brasil

E-mail: iluskasmartins@gmail.com 


\begin{abstract}
Resumo
Introdução: O paciente oncológico requer vários tratamentos, e por isso o trabalho voltado ao mesmo deve ser realizado de forma ampliada. A equipe multiprofissional é formada por médicos, enfermeiros, nutricionistas, psicólogos, e pelo farmacêutico, que está cada vez mais conquistando seu espaço. Objetivo: Conhecer e discutir por meio de um levantamento bibliográfico os avanços da atuação do farmacêutico na oncologia. Metodologia: Se trata de uma revisão de literatura por meio de buscas nas plataformas LILACS, SciELO, MEDLINE, Science Direct e BVS, de artigos e teses publicados no período de 2014 a 2019. Resultados e Discussão: O farmacêutico no âmbito oncológico se torna o principal instrumento para obter a devida qualidade da farmacoterapia individualizada. Sabe-se que em relação à quimioterapia o profissional atua selecionando, adquirindo, armazenando e padronizando os componentes que são primordiais desde o preparo até a dispensação desses antineoplásicos. Mesmo com todo o respaldo e atribuições voltados a profissão farmacêutica, sabe-se que a atuação totalmente satisfatória com atenção farmacêutica em oncologia ainda é um desafio. De acordo com um levantamento do perfil da atuação do farmacêutico em oncologia realizado pela Sobrafo em relação ao total de horas dedicadas a manipulação, é certo dizer que $34,5 \%$ desses profissionais chegam a ficar de 6 horas manipulando e 15,6\% chegam até 8 horas nesse setor. Conclusão: Mesmo com todas as atribuições e avanços do profissional farmacêutico, ainda existe alguns desafios relacionados ao exercício pleno da atenção farmacêutica, como o próprio excesso de tempo no setor de manipulação o que dificulta outras atividades.
\end{abstract}

Palavras-chave: Cuidados farmacêuticos; Farmacêuticos; Oncologia; Câncer; Atenção farmacêutica.

\begin{abstract}
Introduction: The cancer patient requires several treatments, so the work aimed at this patient should be performed in a broad erway. The multiprofessional team is made up of doctors, nurses, nutritionists, psychologists, and the pharmacist, who is increasingly conquering his space. Objective: To know and discus sthrough a bibliographic survey the advance softhe pharmacist's performance in oncology. Methodology: This is a literature review through searches on LILACS, SciELO, MEDLINE, Science Direct and VHL platforms, articles and theses published from 2014 to 2019. Results and Discussion: The oncology pharmacist becomes the main instrument to obtain the proper quality of individualized pharmacotherapy. Regarding chemotherapy, the professional acts by selecting, acquiring, storing and standardizing the components that are paramount from the preparation to the dispensation of these antineoplastics. Even with all the support and attributions focused on the pharmaceutical profession, it is known that the totally satisfactory performance with pharmaceutical attention in oncologyis still a challenge. According to a survey of the profile of the pharmacist's performance in oncology conducted by Sobrafo in relation to the total hours dedicated to manipulation, it is true to say that $34.5 \%$ of these professionals reach 6 hours of manipulation and $15.6 \%$ reach 8 hours in this sector. Conclusion: Even with all the attributions and advances of the pharmaceutical professional, there are still some challenges related to the full exercise of pharmaceutical care, such as the excess of time in the handling sector, which makes other activities difficult.
\end{abstract}

Keywords: Phamaceutical care; Pharmacists; Oncology; Cancer; Pharmaceutical attention.

\title{
Resumen
}

Introducción: El paciente oncológico requiere de varios tratamientos, por lo que el trabajo encaminado a los mismos debe realizarse de forma ampliada. El equipo multiprofesional está formado por médicos, enfermeras, nutricionistas, psicólogos y el farmacéutico, que cada vez va conquistando su espacio. Objetivo: Conocer y discutir a través de un relevamiento bibliográfico los avances del desempeño del farmacéutico en oncología. Metodología: Se trata de una revisión de la literatura mediante búsquedas en las plataformas LILACS, SciELO, MEDLINE, Science Direct y VHL, de artículos y tesis publicados en el período de 2014 a 2019. Resultados y discusión: El farmacéutico en el campo de la oncología se convierte en el principal instrumento para obtener la calidad adecuada de la farmacoterapia individualizada. Se sabe que en relación con la quimioterapia, el profesional actúa seleccionando, adquiriendo, almacenando y estandarizando los componentes que son imprescindibles desde la preparación hasta la dispensación de estos agentes antineoplásicos. Incluso con todo el apoyo y atribuciones dirigidas a la profesión farmacéutica, se sabe que el desempeño plenamente satisfactorio con la atención farmacéutica en oncología sigue siendo un desafío. Según una encuesta sobre el perfil del desempeño del farmacéutico en oncología realizada por Sobrafo en relación al total de horas dedicadas a manipulación, es cierto que el 34,5\% de estos profesionales dedica 6 horas a manipular y el $15,6 \%$ llega hasta las 8 horas. en este sector. Conclusión: Incluso con todas las funciones y avances del profesional farmacéutico, persisten algunos retos relacionados con el ejercicio pleno de la atención farmacéutica, como el exceso de tiempo en el sector de la manipulación, que dificulta otras actividades.

Palabras clave: Cuidado farmacéutico; Farmacéuticos; Oncología; Cáncer; Atención farmacéutica.

\section{Introdução}

A oncologia é a especialidade médica que estuda os tumores, e tem como principal forma de tratamento a quimioterapia, que é a utilização de agentes químicos de forma isolada ou em conjunto, com objetivo curativo ou paliativo, de 
acordo com a extensão da doença, o tipo de tumor e capacidade física do paciente. Além disso, a quimioterapia tem se tornado uma das mais promissoras e importantes maneiras de combater o câncer (Fengler, et al., 2014; Santos, et al., 2018).

A terapêutica de um paciente oncológico requer vários tratamentos combinados, e por isso o trabalho voltado a esse paciente deve ser realizado de forma que atenda todas suas necessidades. Com isso, sabe-se da importância da equipe multiprofissional no acompanhamento do paciente durante todo o tratamento. A equipe é formada por médicos, enfermeiros, nutricionistas, psicólogos, assim também como o profissional farmacêutico, que está cada vez mais conquistando seu espaço neste meio (Lobato et al., 2019).

O Conselho Federal de Farmácia criou em 1996 a resolução 288 que diz: "É atribuição privativa do farmacêutico a competência para o exercício da atividade de manipulação de drogas antineoplásicas e similares nos estabelecimentos de saúde”. Nesta é detalhada a função do profissional de farmácia no exercício da atividade da quimioterapia.

A ANVISA (Agência Nacional de Vigilância Sanitária) confirmou essa resolução em 2004 ao criar a Equipe Multiprofissional da Terapia Antineoplásica- EMTA, estabelecendo assim que qualquer serviço de oncologia, como a quimioterapia, deve obrigatoriamente dispor de médico oncologista ou hematologista, um enfermeiro e um farmacêutico (Sobrafo, 2019).

A atuação do farmacêutico na assistência à saúde tende a evoluir além das funções tradicionais relacionadas aos medicamentos. O Farmacêutico começa a participar ativamente de todas as etapas do processo de tratamento do paciente, onde é capaz de intervir para prevenção de falhas antes da administração de medicamentos, o que consequentemente traz maior segurança ao paciente (Carvalho, 2018).

O farmacêutico trabalha envolvido com os outros profissionais da saúde para resolver e evitar problemas na farmacoterapia, e para isso deve manter-se sempre atualizado sobre a farmacoterapêutica, com a prática farmacêutica e ferramentas que podem ser usadas para acesso à informação. Dessa forma, o farmacêutico deve encontrar e resolver de modo sistematizado e documentado os problemas relacionados ao medicamento que possam surgir durante o tratamento, além de acompanhar o paciente para um atendimento mais seguro (Marques, 2017).

São funções do farmacêutico a seleção, padronização, aquisição e conservação dos medicamentos, insumos farmacêuticos e outros produtos para o estabelecimento de saúde, conferindo sempre se todos os fatores estão de acordo com as normas e exigências pelos órgãos responsáveis. Além disso, o farmacêutico também é responsável por analisar prescrições, verificando se a escolha do fármaco está correta, se é realmente a melhor opção, se faz parte dos protocolos daquele estabelecimento, confere também doses, diluentes e é responsável pela manipulação dos antineoplásicos (SOUZA, et al., 2016).

Diante o exposto, o trabalho teve como objetivo conhecer e discutir por meio de levantamento bibliográfico os avanços da atuação do farmacêutico na oncologia.

\section{Metodologia}

O estudo se trata de uma revisão de literatura, que teve como finalidade apresentar e resumir os estudos já produzidos em relação à profissão farmacêutica no âmbito oncológico, sintetizando as evidências sobre os avanços deste profissional na área, a fim de contribuir para o desenvolvimento dos conhecimentos dessa temática.

A pesquisa seguiu metodologia adaptada de Pereira (2018), com a estratégia de busca eletrônica por artigos, teses, e publicações em revistas pertinentes ao assunto e para isso foram utilizadas as bases de dados: Literatura Latino-Americana e do Caribe em Ciências da Saúde (LILACS), biblioteca eletrônica Scientific Electronic Library Online (SciELO), Sistema Online de Busca e Análise de Literatura Médica (MEDLINE), Science Direct e Biblioteca Virtual em Saúde (BVS). Os 
descritores utilizados para a busca foram: "Cuidados Farmacêuticos", "Farmacêuticos", "Oncologia", "Câncer", "Atenção Farmacêutica".

Como critérios de inclusão foram utilizados trabalhos em formato de artigo, revisão, dissertação e teses publicadas no período de 2014 a 2019, que abordavam sobre o profissional farmacêutico no âmbito oncológico, em inglês, português ou espanhol. Já os trabalhos excluídos para pesquisa foram aqueles que após leitura não se referiam ao objetivo principal da pesquisa, trabalhos datados de anos anteriores e com duplicidade.

\section{Resultados e Discussão}

A atuação do farmacêutico na área de oncologia começou a ser concebido a partir da década de 90 com base em normas internacionais e nas experiências de trabalhos executados em grandes centros hospitalares a partir de 1946 com publicações de estudos clínicos, onde começaram a identificar riscos trabalhistas e ambientais, até mesmo a exigência de rigorosa técnica asséptica para a manipulação de medicamentos para uso parenteral, onde a falta de conhecimento é uma causa importante de acidentes no ambiente de trabalho (CFF, 2019).

Por apresentarem índice terapêutico estreito, eles possuem alto potencial para causar eventos adversos, sendo que tanto a resposta terapêutica quanto sua toxicidade são relacionadas à concentração plasmática do fármaco e seu tempo de permanência no organismo. Dessa forma, são considerados medicamentos potencialmente perigosos, necessitando de alta vigilância em todas as etapas da sua utilização (ISMP, 2014).

De acordo com Souza et al., (2017) O controle criterioso das dosagens gerou a necessidade de estabelecer rotinas e adequar áreas específicas para essas tarefas, exigindo um maior aperfeiçoamento técnico do farmacêutico. A maioria desses profissionais não teve acesso ao conhecimento sobre biossegurança em oncologia na graduação, apesar de ter cursado a grade curricular generalista, evidenciando o despreparo do farmacêutico para atuação nesse âmbito.

O farmacêutico no âmbito oncológico se torna o principal instrumento para obter a devida qualidade da farmacoterapia individualizada. Sabe-se que em relação à quimioterapia o profissional atua selecionando, adquirindo, armazenando e padronizando os componentes que são primordiais desde o preparo até a dispensação desses antineoplásicos (Santos et al., 2018).

Em 2012 o Conselho Federal de Farmácia (CFF) por meio de nota técnica emitiu um parecer sobre a atuação do farmacêutico na manipulação de antineoplásicos, objetivando alinha conceitos e entendimentos acerca do tema com os conselhos regionais de farmácia (CRFs) onde consta que a manipulação de medicamentos antineoplásicos é ato privativo intransferível e indelegável ao farmacêutico. Portanto nenhum outro profissional de saúde quer seja ele nível superior ou de nível médio, pode realizar esse ato nem mesmo sob supervisão do farmacêutico.

O farmacêutico atua também analisando a prescrição e seus componentes presentes em relação à quantidade, qualidade, compatibilidade, estabilidade e suas interações com objetivo de melhorar a adesão a terapia e consequentemente trazer segurança ao paciente (Lobato et al., 2019).

\subsection{Avanços da profissão farmacêutica no âmbito oncológico}

A RDC n 220/2004 do Conselho Federal de Farmácia regulamenta e preconiza as atribuições e funções de cada membro da equipe multiprofissional em terapia antineoplásica - EMTA, as quais precisam ser claramente definidas para que se tenha a eficiência esperada. Além desta, o Farmacêutico conta ainda com a resolução 288/1996, que trata especificamente das atribuições deste profissional. 
O farmacêutico vem ampliando sua área de atuação no âmbito da oncologia desde a década de 90, onde o Conselho Federal de Farmácia estabelece por meio da RDC n 288/1996, a competência legal para o exercício da manipulação de drogas antineoplásicas pelo farmacêutico. Segundo o Conselho Federal de Farmácia são atribuições do farmacêutico:

Art. $1^{\circ}$ - É atribuição privativa do farmacêutico a competência para o exercício da atividade de manipulação de drogas antineoplásicas e similares nos estabelecimentos de saúde;

Art. $2^{\circ}$ - No exercício da atividade de quimioterapia nos estabelecimentos de saúde, caberá ao farmacêutico;

I. Selecionar, adquirir, armazenar e padronizar os componentes necessários ao preparo dos antineoplásicos;

II. Avaliar os componentes presentes na prescrição médica, quanto a quantidade, qualidade, compatibilidade, estabilidade e suas interações;

III. Proceder a formulação dos antineoplásicos segundo prescrição médica, em concordância com o preconizado em literatura;

IV. Manipular drogas antineoplásicas em ambientes e condições assépticos, e obedecendo critérios internacionais de segurança;

V. Orientar, supervisionar e estabelecer rotinas nos procedimentos de manipulação e preparação dos antineoplásicos;

VI. Preencher adequadamente o rótulo de cada unidade de antineoplásico preparado, assinar e carimbar, identificando o nome do cliente da terapêutica, a quantidade de cada componente adicionado, bem como efetuar as devidas recomendações para sua estabilidade e administração;

VII. Determinar o prazo de validade para cada unidade de antineoplásico de acordo com as condições de preparo e características da substância;

VIII. Assegurar o controle de qualidade dos antineoplásicos após o preparo até a administração;

IX. Registrar cada solução de antineoplásico preparado em livro de registro exclusivo com termo de abertura, onde constará: data do preparo, nome completo, número do prontuário do paciente e localização, número sequencial de preparo, diagnóstico, protocolo de referência, quantidade preparada, concentrações do produto;

$\mathrm{X}$. Assegurar destino seguro para os resíduos dos antineoplásicos;

XI. Assegurar a observância das normas de segurança individuais e coletivas para a manipulação de antineoplásicos recomendados em nível nacional e internacional;

XII. Informar periodicamente, ou quando solicitado, o custo de cada componente de solução após o preparo;

XIII. Compor a equipe multidisciplinar nas visitas aos clientes submetidos ao tratamento com antineoplásicos;

XIV. Participar das reuniões, discussões de casos clínicos e atividades didáticas e científicas da equipe multidisciplinar;

XV. Possibilitar estágios supervisionados à farmacêuticos e acadêmicos de farmácia;

XVI. O farmacêutico deverá dispor de dados quanto a qualidade destes produtos, sobretudo garantindo os seguintes parâmetros: solubilidade, estabilidade, homogeneidade, viscosidade, osmolaridade, esterilidade, teor e pureza;

XVII. Participar, desenvolver, elaborar pesquisas de antineoplásicos, não só na área de saúde, bem como na área industrial;

XVIII. Participar e atuar em toda divulgação técnica científica vinculada ao marketing do suporte quimioterápico.

Em 2001 foi criada a Sociedade Brasileira de farmacêuticos em Oncologia - SOBRAFO, a qual fomenta o fortalecimento da classe, dando suporte técnico e científico para os profissionais da área (SOBRAFO, 2019).

A SOBRAFO tem como objetivos estratégicos:

- Promover do desenvolvimento e adoção de práticas seguras e de qualidade envolvendo medicamentos para tratamento e suporte do paciente com câncer; 
- Divulgar conhecimento técnico-científico e desenvolver ações para o aprimoramento dos serviços farmacêuticos na linha de cuidado em oncologia;

- Estimular a adoção de práticas para melhoria contínua da gestão de serviços farmacêuticos oncológicos;

- Incentivar e apoiar ações de ensino, treinamento e pesquisa para qualificação de seus membros atuantes na Assistência Farmacêutica em Oncologia - AFO;

- Organizar e propor políticas e diretrizes para o pleno desenvolvimento da AFO no sistema de saúde, na perspectiva dos setores público e privado e;

- Promover articulação com sociedades cientifica e organizações nacionais e internacionais relacionadas ao contexto da atenção oncológica, visando aprimorar a AFO no Brasil e nos demais países da América Latina.

Recentemente o Conselho Federal de Farmácia publicou a RDC nº640 de 2017, que dá nova redação ao artigo $1^{\circ}$ da Resolução/CFF nº 623/16, estabelecendo titulação mínima para a atuação do farmacêutico em oncologia, a mesma relata:

"Art. $1^{\circ}$ - É atribuição privativa do farmacêutico o preparo dos antineoplásicos e demais medicamentos que possam causar risco ocupacional ao manipulador (teratogenicidade, carcinogenicidade e/ou mutagenicidade) nos estabelecimentos de saúde públicos ou privados.

$\S 1^{\circ}$ - Para o exercício de atividades de preparo dos antineoplásicos e demais medicamentos na oncologia, deverá o farmacêutico atender a pelo menos um dos seguintes critérios, validado pelo Conselho Regional de Farmácia de sua jurisdição:

a) ser portador de título de especialista emitido pela Sociedade Brasileira de Farmacêuticos em Oncologia (Sobrafo);

b) ter feito residência na área de Oncologia;

c) ser egresso de programa de pós-graduação lato sensu reconhecido pelo Ministério da Educação (MEC) relacionado à farmácia oncológica; d) ter atuado por 3 (três) anos ou mais na área de oncologia, o que deve ser comprovado por meio de Carteira de trabalho e Previdência Social (CTPS) ou de contrato e declaração do serviço, com a devida descrição das atividades realizadas e do período de atuação.

$\S 2^{\circ}$ - Aos farmacêuticos que atuam e aos que estão interessados em atuar nesta área dar-se-á o prazo de 36 (trinta e seis) meses para adequação de currículo e titulação, no que se refere ao parágrafo anterior."

A atuação do profissional farmacêutico em oncologia é presente em quase todos os serviços de quimioterapia do Brasil, e mesmo tendo iniciado sua atuação apenas na manipulação e gerenciamento de quimioterápicos, o profissional vem se tornando cada vez mais essencial para garantia da qualidade dos procedimentos (Fengler et al., 2014).

\subsection{Atenção farmacêutica em oncologia atualmente}

A atenção farmacêutica focada no paciente surge na intenção de melhoria da qualidade do atendimento e do processo de utilização de medicamentos para que alcance resultados cada vez mais vantajosos (Kazmirczak, 2016).

Segundo Carvalho (2018), a Organização mundial de Saúde - OMS em 1994 estendeu a atenção farmacêutica para toda comunidade reconhecendo o farmacêutico como dispensador de atenção à saúde, devendo participar ativamente em conjunto com os outros membros da equipe na prevenção de doenças e promoção a saúde.

O farmacêutico deve complementar os serviços médicos com objetivo de informar os pacientes sobre a real finalidade do medicamento e da terapia de suporte utilizada, os possíveis efeitos adversos e interações medicamentosas, dando ênfase sempre para ocorrência dessas reações e formas de como podem ser evitadas (Carvalho, 2018).

Para o Conselho Federal de Farmácia por meio da RDC n³57, de 20 de abril de 2001 que aprova o regulamento técnico das Boas Práticas de Farmácia, a atenção farmacêutica é caracterizada por atitudes do farmacêutico, as quais o paciente é principal beneficiário. 
O farmacêutico juntamente aos outros profissionais atua mais efetivamente, gerando maior segurança e eficácia da farmacoterapia, por meio da identificação, da resolução e da prevenção dos problemas relacionados a medicamentos - PRM (Aguiar, et al., 2018).

Durante o tratamento oncológico os pacientes podem ficar sujeitos a sofrerem algum erro de medicação principalmente devido a erros de prescrição, e uns dos principais serviços de oncologia realizado pelo farmacêutico é a análise da prescrição e consequente a intervenção farmacêutica (Barbosa, 2018).

Estes erros podem ser relacionados à ilegibilidade da prescrição, ao uso de abreviaturas e até mesmo erros relacionados a características do próprio paciente, como a falta de ajuste de dose do antineoplásico por conta de alteração na função renal ou até mesmo uma alergia. O sucesso terapêutico é alcançado quando esses erros são identificados de forma precoce, aumentando assim a probabilidade de sucesso do tratamento do paciente. (Ranchon et al., 2012).

O farmacêutico clínico que atua na área oncológica deve ser devidamente treinado, pois ele também será responsável pela gestão e prevenção de problemas relacionados aos medicamentos, fator importantíssimo devido ao alto custo, alta toxicidade e baixo índice terapêutico dos antineoplásicos, além de ser capaz de lidar com pacientes com estado de saúde muito frágil (Hopa, 2014).

O farmacêutico deverá fornecer educação aos outros profissionais da equipe, sobre os medicamentos e protocolos institucionais, identificando os possíveis problemas relacionados ao medicamento, como dose inapropriada, adesão aos fármacos orais e até mesmo a educação voltada ao paciente (Barbosa, 2018).

Mesmo com todo o respaldo e atribuições voltados a profissão farmacêutica neste âmbito, sabe-se que a atuação totalmente satisfatória com atenção farmacêutica em oncologia ainda é um desafio. De acordo com um levantamento do perfil da atuação do farmacêutico em oncologia realizado pela Sobrafo em relação ao total de horas dedicadas a manipulação é certo dizer que 34,5\% desses profissionais chegam a ficar de 6 horas manipulando e 15,6\% chegam até 8 horas (SOBRAFO, 2017).

Com isso é perceptível que além das implicações corriqueiras para realização da atenção farmacêutica em si, a própria falta de tempo também se torna uma barreira para tal prática.

\section{Conclusão}

Com base nos resultados apontados na presente pesquisa é possível concluir que o profissional farmacêutico tem seu perfil tradicional alterado, podendo agora juntamente com a equipe multiprofissional lidar com o paciente como foco de seu trabalho, conseguindo assim desempenhar função importantíssima na melhoria da qualidade de vida de seu paciente.

Porém, mesmo com todas as atribuições e avanços do profissional farmacêutico, o mesmo ainda possui alguns desafios relacionados ao exercício pleno da atenção farmacêutica, como o próprio excesso de tempo no setor de manipulação, o que torna menor o tempo hábil para as atividades de atenção farmacêutica voltadas mais diretamente ao paciente.

\section{Referências}

Aguiar, K. S. (2018). Segurança do paciente e o valor da intervenção farmacêutica em um hospital oncológico. Einstein - São Paulo - SP. 16(1), 1-7.

Azevedo, E. A. et al. (2014). Antineoplásicos parenterais: erros de medicação, riscos e práticas seguras na utilização. Instituto para práticas seguras no uso de medicamentos. Belo Horizonte - MG. 3(3).

Barbosa, C. R. (2018). Farmacêutico clínico em oncologia: contribuição efetiva para segurança do paciente. Dissertação de Mestrado. Universidade Estadual de Campinas. Campinas- SP.

Brasil. (2004). Agência Nacional de Vigilância Sanitária. Resolução - RDC $N^{o} 220$, de 21 de setembro de 2004 . Aprova o regulamento técnico de funcionamento dos Serviços de Terapia Antineoplásica. http://portal.anvisa.gov.br/wps/wcm/connect/a5d8d680474597419facdf3fbc4c6735/RDC+N\%C2\%BA+220-2004.pdf?MOD=AJPERES.

Brasil. (1996). Conselho federal de Farmácia. Resolução $N^{\circ} 288$ de 21 de março de 1996. Dispõe sobre a competência legal para o exercício da manipulação de drogas antineoplásicas pelo farmacêutico. http://www.cff.org.br/userfiles/file/resolucoes/288.pdf. 
Research, Society and Development, v. 10, n. 9, e9210915794, 2021

(CC BY 4.0) | ISSN 2525-3409 | DOI: http://dx.doi.org/10.33448/rsd-v10i9.15794

Brasil. (2001). Conselho Federal de Farmácia. Resolução N 357 de 20 de abril de 2001. Aprova o regulamento técnico das Boas Práticas de Farmácia. http://www.cff.org.br/userfiles/file/resolucoes/357.pdf.

Brasil. (2017). Conselho Federal de Farmácia. Resolução $N^{\circ} 640$ de 27 de abril de 2017. Dá nova redação ao artigo $1^{\circ}$ da Resolução/CFF $n^{\circ}$ 623/16, estabelecendo titulação mínima para a atuação do farmacêutico en emcologia. http://pesquisa.in.gov.br/imprensa/jsp/visualiza/index.jsp?data=08/05/2017\&jornal=1\&pagina=121\&totalArquivos=128.

Brasil. (2019). Conselho Federal de Farmácia. Referenciais mínimos para o reconhecimento de cursos livres para a especialização profissional farmacêutica, sem caráter acadêmico, em oncologia. https://cff-br.implanta.net.br/portaltransparencia/\#publico/Listas?id=2068835e-f6e0-451e-956c-35103fc0c9af.

Carvalho, G. A. C. (2018). Impacto das intervenções do farmacêutico clínico no cuidado de pacientes oncológicos: uma revisão sistemática. Dissertação de pós-graduação. Universidade Federal De Sergipe. São Cristovão - SE.

Fengler, A. C. et al. (2014). A atuação do farmacêutico no tratamento do paciente oncológico. Salão do conhecimento. Ijuí-RS.

Hopa. (2014). Hematology/Oncology Pharmacy Association. The role ofhematology / oncology pharmacists. http://www.hoparx.org/images/hopa/advocacy/Issue-Briefs/HOPA.

Kazmirczak, A. (2016). Contribuições da assistência farmacêutica para o paciente oncológico. Trabalho de Conclusão de Curso. Universidade Regional Do Noroeste Do Estado Do Rio Grande Do Sul. Ijuí - RS

Lobato, L. C., et al. (2019). Cuidados farmacêuticos no tratamento oncológico: uma revisão integrativa da literatura. Conexão Ciência. Formiga - MG, 14(1), 31-38.

Marques. O. H. M. S. (2017). Atuação do farmacêutico no âmbito da oncologia. Trabalho de Conclusão de Curso. Universidade Estadual da Paraíba. Campo Grande - PB.

Pereira A. S. et al. (2018). Metodologia da pesquisa científica. [free e-book]. Santa Maria/RS. Ed. UAB/NTE/UFSM.

Ranchon F, et al. (2012). Predictors of prescription errors involving anticancer chemothera pyagents. Eur J Cancer. 48(8), 1192-199.

Santos, S. L. F. et al. (2018). Evidências do cuidado farmacêutico na prática clínica da oncologia. Rev. FacCiêncMéd. Sorocaba. 20(2), 77-81.

Sobrafo. (2017). Perfil da atuação do farmacêutico em oncologia https://sobrafo.org.br/wpcontent/uploads/2019/03/infografico_perfil_farmaceutico_final.pdf. 2017

Sobrafo. (2019). Espaço do estudante. https://sobrafo.org.br/

Sousa, M. et al. (2016). Atuação do farmacêutico hospitalar na oncologia. Boletim Informativo Geum, 7(1), 54-63.

Souza, D. F. et al. (2017). Biossegurança em oncologia e o profissional farmacêutico: análise de prescrição e manipulação de medicamentos antineoplásicos. Revista Baiana saúde pública, Bahia-BA, 40(4). 